\title{
Previsão e interação dos preços da celulose brasileira nos mercados interno e externo
}

\author{
Forecast and interactions of the brazilian cellulose prices in the internal and \\ external markets
}

\author{
Bianca Reichert ${ }^{\mathrm{I}}$, Adriano Mendonça Souza ${ }^{\mathrm{II}}$
}

\begin{abstract}
Resumo
A produção e a exportação de celulose são componentes importantes da economia no país. O objetivo desta pesquisa foi prognosticar o preço nos mercados interno e externo da celulose brasileira e avaliar a interferência entre o preço médio da celulose vendida em atacado e o preço médio da celulose exportada pelo Brasil. O estudo utilizou dados oriundos do Informativo Florestal do Centro de Estudos Avançados em Economia Aplicada (CEPEA), coletados entre junho de 2008 a março de 2018. Os modelos Autorregressivos Integrados de Médias Móveis (ARIMA) foram utilizados para prognosticar o preço da celulose em atacado e exportada pelo Brasil e, para analisar a inter-relação dessas variáveis, foi aplicado modelo Vetor Autorregressivo (VAR). Observou-se que o preço da celulose em atacado e da celulose exportada sofrem oscilações em períodos semelhantes, devido à relação direta com a cotação do dólar e com as crises financeiras nos países importadores. O modelo de melhor acurácia para prognosticar o preço da celulose em atacado foi o modelo ARIMA $(1,1,0)$ enquanto o modelo ARFIMAX $\left(1, d^{\star}, 0\right)$ obteve o melhor desempenho para prognosticar o preço da celulose exportada. A partir da modelagem VAR, verificou-se a existência de inter-relações entre as variáveis, as quais transpareceram o forte impacto do preço da celulose em atacado sobre o preço da celulose exportada pelo Brasil. Assim, as metodologias empregadas foram eficazes para prognosticar e analisar as inter-relações entre as variáveis.
\end{abstract}

Palavras-chave: Séries temporais; Modelos ARIMA; Vetor autorregressivo; Previsão do preço

\begin{abstract}
The production and export of cellulose are important components of the Brazilian economy. The aim of this research was to predict the domestic and foreign prices of the Brazilian cellulose and to evaluate the interference between its average price sold at wholesale and exported by Brazil. The study focused on data from the Forestry Newsletter of the Center for Advanced Studies on Applied Economics (CEPEA), collected from June 2008 to March 2018. The Autoregressive Integrated Moving Average (ARIMA) models were used to predict the wholesale and export price of cellulose, while the Vector Autoregressive (VAR) model was applied to analyze the inter-relation of these variables. It was observed that the price of wholesale and exported celluloses vary in similar periods, due to the direct relationship with the dollar quotation and with the financial crises in the importing countries. The most accurate model adjusted to predict the wholesale cellulose price was the ARIMA model $(1,1,0)$, while ARFIMAX model $\left(1, d^{*}, 0\right)$ obtained the best performance to predict exported cellulose price. Based on the VAR model, there was a correlation between variables, which means that wholesale cellulose price has a strong impact on exported cellulose price. Thus, the methodologies used were effective to predict and analyze the inter-relationships between the variables.
\end{abstract}

Keywords: Time series; ARIMA models; Autoregressive vector; Price forecast

Engenheira de Produção, Doutoranda do Curso de Pós-Graduação em Engenharia de Produção, Centro de Tecnologia, Universidade Federal de Santa Maria, Av. Roraima, 1000, CEP 97105-900, Santa Maria (RS), Brasil. bianca.reichert@hotmail.com (ORCID: 0000-0003-1741-5440)

II Engenheiro de Produção, Dr., Professor do Departamento de Estatística, Centro de Ciências Naturais e Exatas, Universidade Federal de Santa Maria, Av. Roraima, 1000, CEP 97105-900, Santa Maria (RS), Brasil. amsouza@smail.ufsm.br (ORCID: 0000-0002-1562-2246) 


\section{Introdução}

A celulose é o principal composto utilizado para a fabricação de papéis e papelão. O seu processo de obtenção consiste na extração e no tratamento de fibras vegetais, as quais provêm de árvores coníferas e folhosas, como das espécies dos gêneros Pinus e Eucalyptus, respectivamente (CORREA, 2014; MONTENEGRO, 2018).

A importância da indústria da celulose para a economia brasileira reflete no PIB do país, a qual possui representatividade de $6,9 \%$. Além do impacto na economia interna, a celulose brasileira se destaca nos mercados externos, ao movimentar cerca de $5,2 \%$ do total das exportações brasileiras em 2018, o equivalente a US $\$ 12,5$ bilhões (INDÚSTRIA BRASILEIRA DE ÁRVORES, 2018). Os principais países importadores da celulose brasileira são China, Estado Unidos, Alemanha e Itália, os quais consomem aproximadamente $62 \%$ do total da celulose produzida pelo país (MOURA et al., 2018).

Em relação ao agronegócio, a exportação de celulose ocupa a quarta posição, quando comparada com as exportações de carne suína, soja e algodão. Projeções apontam que, até o ano de 2028 , mais de $70 \%$ da celulose produzida no Brasil será exportada para outros países (BRASIL, 2018).

Dentre as maiores indústrias papeleiras do país, destacam-se a Suzano e a Klabin, as quais possuem como fator competitivo a qualificação das suas equipes e o desenvolvimento de florestas altamente produtivas (CELULOSE ONLINE, 2017; O PAPEL, 2019). Porém, como as empresas estão voltadas para o mercado externo, a variação no preço de venda da celulose e a oscilação cambial do dólar influenciam diretamente o lucro operacional dessas empresas (SANTOS et al., 2019). Essas variações podem impactar até $80 \%$ do resultado financeiro das empresas, uma vez que o lucro terá efeito negativo se o preço da celulose e o dólar apresentarem comportamentos decrescentes (HORA; VIDAL, 2014; ROSSATO et al., 2018; INVESTING, 2019).

As empresas papeleiras também exercem grande influência social nas comunidades em que estão inseridas (SANTOS; ARAÚJO; GITAHY, 2016). Em estudos realizados por Oliveira, Pereira e Nascimento (2018) verificou-se expansão urbana, aumento da capitalização das comunidades, aumento populacional e maior oferta de emprego nas cidades onde as empresas instalaram suas indústrias.

Soares et al. (2015) enfatizaram a importância da realização de estudos sobre o preço da celulose, dado que essa informação auxilia na definição de estratégias de venda do produto para diferentes países importadores. Para tanto, os modelos Autorregressivos Integrados de Médias Móveis (ARIMA) são úteis para análise e previsão do preço da celulose, pois baseiam-se em valores passados e suas correlações para predizer os valores futuros (BOX; JENKINS, 1970). A metodologia de Box e Jenkins apresenta aplicações para variáveis do setor de celulose e papel, como em estudos realizados por Ersen, Akyüz e Bayram (2019), os quais realizaram a prognose dos níveis de exportações e importações de papel na Turquia.

A metodologia de redes neurais também foi utilizada por Santos et al. (2019) para realizar a previsão de indicadores econômico-financeiros das empresas papeleiras brasileiras, na qual o teste de Toda-Yamamoto foi aplicado para verificar dependências entre essas variáveis. Além de testes, as relações de dependências também podem ser verificadas por meio do modelo Vetor Autorregressivo (VAR) (RAMSER et al., 2019).

A partir disso, o problema de pesquisa consiste em verificar se há interferência entre os preços da celulose brasileira praticados nos mercados interno e externo. Assim, o objetivo da pesquisa foi prognosticar o preço da celulose brasileira nos mercados interno e externo, por meio dos modelos da classe geral ARIMA, além de avaliar as interações entre esses mercados por meio da modelagem VAR. 


\section{Material e método}

\section{Coleta de dados}

As variáveis analisadas foram o preço médio da celulose em atacado (US\$/ton.) e o preço médio da celulose exportada pelo Brasil (US\$/ton.). Cada série contém 118 observações mensais referentes ao intervalo de junho de 2008 a março de 2018. Os dados são oriundos do Informativo Florestal do Centro de Estudos Avançados em Economia Aplicada (CEPEA) da Universidade de São Paulo (USP).

\section{Modelagem ARIMA}

Para prognosticar o comportamento do preço da celulose, foram utilizados os modelos Autorregressivos Integrados e de Médias Móveis (ARIMA), cuja modelagem só pode ser aplicada em séries estacionárias com observações autocorrelacionadas (BOX; JENKINS, 1970). Como etapa inicial, utilizou-se o gráfico da série original para identificar comportamentos de tendência e/ ou sazonalidade na série. A estacionariedade foi determinada pelos testes de raízes unitárias DickeyFuller Aumentado (ADF) e Kwiatkowski-Phillips-Schmidt-Shin (KPSS) (KWIATKOWSKI et al., 1922; DICKEY; FULLER, 1981). Nos casos em que a série não apresentou estacionariedade, foi necessário diferenciá-la até alcançar a estabilidade (SOUZA, 2016).

Os gráficos das funções de autocorrelação (FAC) e de autocorrelação parcial (FACP) foram utilizados para verificar a autocorrelação das observações. Essas funções também auxiliaram na identificação do filtro (AR, MA, ARMA, ARIMA, SARIMA, ARFIMA ou ARMAX) e da ordem do modelo a ser utilizado na etapa de estimação (BOX; JENKINS; REINSEL, 1994).

O modelo ARIMA $(p, d, q)$ baseia-se na ideia de que as observações possuem uma ligação, a qual influencia o comportamento futuro da série e, assim, podem-se predizer os valores futuros. A equação do modelo geral ARIMA é dada por:

$$
\phi(B) \Delta^{d} X_{t}=\theta(B) \alpha_{t}
$$

Em que: $B$ = operador de retardo; $\Delta$ = operador de diferenciação; $d=$ ordem de diferenciação; $\phi=$ parâmetro da parte autorregressiva; $\theta$ = parâmetro da parte de médias móveis; $\alpha=$ resíduo; $\mathrm{t}=$ tempo; $X=$ variável em análise.

Uma das variações da modelagem ARIMA consiste na integração fracionária: o modelo ARFIMA. Esse modelo baseia-se na aplicação de diferenças não inteiras (MORETTIN, 2016). Os parâmetros do modelo são $p, d^{*}, q$, em que $d^{*}$ representa a diferença fracionária, a qual deve ser um valor entre $-0,50<d^{*}<0,50$. A equação que representa o modelo $\operatorname{ARFIMA}\left(p, d^{*}, q\right)$ é:

$$
\phi(B) \Delta^{d *} X_{t}=\theta(B) \alpha_{t}
$$

Em que: $B=$ operador de retardo; $\Delta=$ operador de diferenciação; $d^{*}=$ ordem de integração fracionária; $\phi=$ parâmetro da parte autorregressiva; $\theta=$ parâmetro da parte de médias móveis; $\alpha=$ resíduo; $\mathrm{t}=$ tempo; $X=$ variável em análise. 
Outra variação do ARIMA, o modelo autorregressivo de médias móveis com variável exógena (ARMAX), capta o efeito de uma variável externa à série temporal analisada (GUSTIN; MCLEOD; LOMAS, 2018). No modelo $\operatorname{ARMAX}(p, q, y)$, adiciona-se um parâmetro na equação geral, o qual representa o efeito da variável exógena $\left(Y_{t}\right)$, conforme demonstrado na equação a seguir:

$$
\phi(B) \Delta^{d} X_{t}=\theta(B) \alpha_{t}+\phi(B) \Delta^{d} Y_{t}
$$

Em que: $X=$ série de dados em análise; $B=$ operador de retardo; $\Delta=$ operador de diferenciação; $d=$ ordem de diferenciação; $\phi$ = parâmetro que representa a parte autorregressiva; $\theta$ = parâmetro da parte de médias móveis; $\alpha=$ resíduo; $Y=$ série de dados da variável exógena; $\mathrm{t}$ = tempo.

Após o ajuste dos modelos, realizou-se a análise dos resíduos $(\alpha)$, os quais deveriam apresentar valores não autocorrelacionados e serem normalmente distribuídos com variância constante e média igual a zero. Essas características indicam que o resíduo segue um processo do tipo Ruído Branco $\alpha_{t} \approx \mathrm{N}\left(0, \sigma^{2}\right)$ (BUENO, 2015).

A seleção dos modelos concorrentes, dentre os que apresentaram resíduos do tipo Ruído Branco, baseou-se nos critérios de informação Akaike (AIC) e Bayesiano (BIC). Os melhores modelos ajustados são os que apresentam valores mínimos para essas estatísticas (AKAIKE, 1974). Esses critérios são calculados conforme:

$$
\begin{aligned}
& A I C=T \ln (S Q R)+2 p \\
& B I C=T \ln (S Q R)+p \ln (T)
\end{aligned}
$$

Em que: $T$ = número de observações; $S Q R=$ soma dos quadrados dos resíduos; $p$ = número de parâmetros que foram estimados no modelo; $l n=$ logaritmo natural.

Os critérios AIC e BIC penalizam os modelos, pois consideram em sua estatística o número de parâmetros estimados. Isto é, quanto maior for o número de parâmetros $p$ de um modelo, maior será a penalização nos resultados do AIC e BIC (BOZDOGAN, 1987).

\section{Estatísticas de previsão}

Com o objetivo de avaliar e selecionar o melhor modelo para prognosticar o preço da celulose, estimaram-se as estatísticas de previsão com base nos erros de previsões (SOUZA et al., 2011). As principais estatísticas utilizadas foram: Raiz do Erro Quadrático Médio (RMSE); Erro Percentual Médio Absoluto (MAPE); estatística U-Theil.

$$
\begin{aligned}
& \text { RMSE }=\sqrt{\frac{1}{n} \sum\left(X_{t}-\hat{X}_{t}\right)^{2}} \\
& M A P E=\frac{1}{n} \sum\left|\frac{X_{t}-\hat{X}_{t}}{X_{t}}\right| * 100 \\
& U-\text { Theil }=\frac{\sqrt{\sum_{t=1}^{N}\left(X_{t}-\hat{X}_{t}\right)^{2}}}{\sqrt{\sum_{t=1}^{N}\left(X_{t}-X_{t-1}\right)^{2}}}
\end{aligned}
$$

Em que: $X_{t}=$ observação real no instante $t ; \hat{X}_{t}=$ previsão no instante $t ; t=$ tempo. 
As estatísticas de previsão auxiliam na escolha do melhor modelo, pois o modelo que apresentar os menores valores para essas estatísticas demonstrará o melhor desempenho ao realizar a previsão dos valores em análise (SOUZA, 2016). Após escolhido o modelo, elaborou-se o gráfico dos valores reais, dos valores ajustados e dos resíduos da modelagem, com o intuito de identificar se o modelo é capaz de captar os movimentos e oscilações da série original e realizar a sua previsão.

\section{Modelo VAR}

De modo a analisar as inter-relações entre as variáveis, foi estimado um modelo de Vetor Autorregressivo (VAR), com o objetivo de verificar as possíveis relações dinâmicas que ocorrem entre as variáveis em um determinado intervalo de tempo (LÜTKEPOHL, 1991; MADDALA, 1992; CHAREMZA; DEADMAN, 1997). Além disso, para aumentar a acurácia das estimativas do modelo, estimou-se o modelo VAR com $p$ defasagens (HAMILTON, 1994). O modelo VAR (p) genérico é dado por:

$$
X_{t}=v+\varphi_{1} X_{t-1}+\cdots+\varphi_{p} X_{t-p}+\alpha_{t}
$$

Em que: $X=$ um vetor; $\phi=$ matriz dos coeficientes; $v=$ vetor dos interceptos; $\alpha=$ resíduo; $\mathrm{t}=$ tempo; $p=$ número de defasagens.

Para analisar as inter-relações e as variações geradas em cada uma das séries, utilizaramse a Função Impulso de Resposta e a Decomposição da Variância. O Impulso Resposta consiste em aplicar um choque nos resíduos de uma série e verificar o impacto na outra (RAMSER et al., 2019). O choque é aplicado no período $t$ e transferido para os períodos, $t+1, t+2, \ldots, t+\mathrm{i}$ (SENNA; SOUZA, 2016). A análise da Decomposição da Variância possibilita identificar a dinâmica das variáveis ao longo do tempo, por meio da separação da variância dos resíduos das previsões para cada variável (MARGARIDO; TUROLLA; FERNANDES, 2002).

\section{Resultados e discussão}

Os valores médios do preço da celulose comercializada nos mercados interno e externo podem ser observados na Figura 1, bem como o comportamento das séries no intervalo entre junho de 2008 e março de 2018.

Verifica-se que o preço médio da celulose exportada é menor que o preço médio da celulose comercializada em atacado (Figura 1). Isso reflete a realidade do mercado externo, na qual, muitas vezes, o preço é estipulado e defasado pelos países importadores. Entretanto, o custo de produção da celulose brasileira é menor, em comparação com o de outros países produtores, o que aumenta a competitividade do Brasil nas exportações de celulose e seus derivados (HORA; RIBEIRO; MENDES, 2018).

Além disso, observa-se que as oscilações positivas e negativas ocorrem nas séries em períodos semelhantes, o que indica uma possível correlação entre esses valores, pois variações em índices econômicos afetam da mesma forma os mercados interno e externo da celulose brasileira (BATISTA, 2019).

Antes de aplicar a modelagem ARIMA e suas derivações, foram elaborados os gráficos da FAC e FACP, Figuras 2 e 3, com o intuito de analisar a estacionariedade das séries e a existência de autocorrelação entre as observações. 
Figura 1 - Preço médio mensal da celulose brasileira em atacado e exportada no período de junho de 2008 a março de 2018

Figure 1 - Monthly average price of wholesale and exported Brazilian cellulose from June 2008 to March 2018

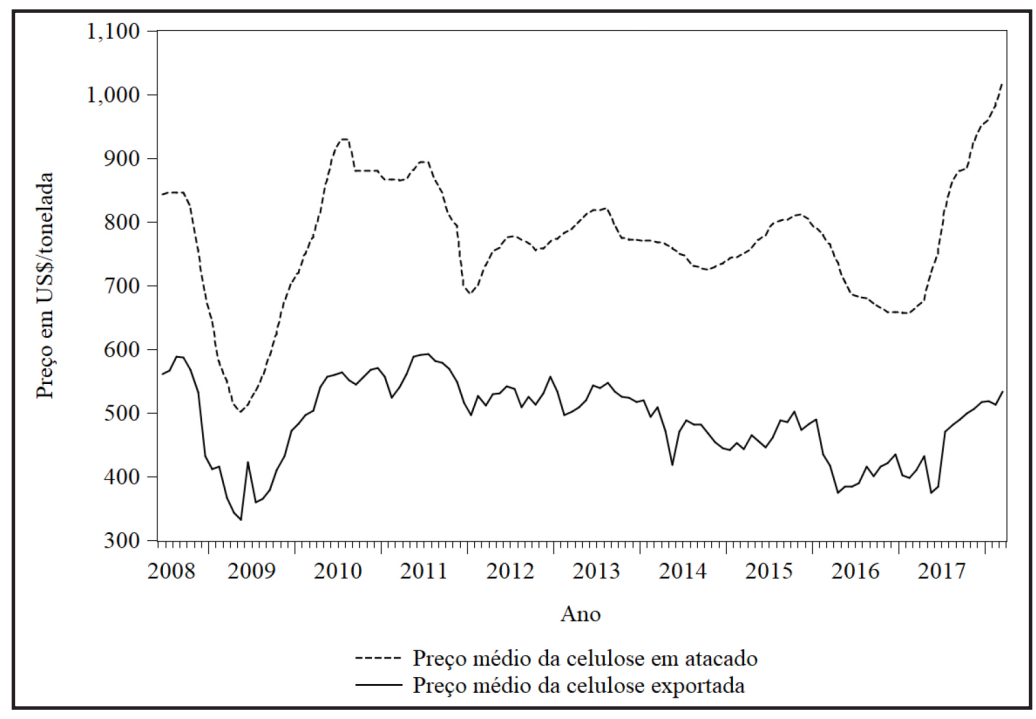

Fonte: Autores (2019)

Figura 2 - Gráfico da Função de Autocorrelação e Função de Autocorrelação Parcial do preço médio da celulose brasileira em atacado, referente ao período de junho de 2008 a março de 2018

Figure 2 - Autocorrelation and Partial Autocorrelation Function chart of the average wholesale Brazilian cellulose price from June 2008 to March 2018

\begin{tabular}{|c|c|c|c|c|c|c|c|c|}
\hline \multicolumn{2}{|c|}{ Autocorrelation } & \multicolumn{2}{|c|}{ Partial Correlation } & & $\mathrm{AC}$ & PAC & Q-Stat & Prob \\
\hline I & 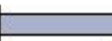 & 1 & & 1 & 0.940 & 0.940 & 107.03 & 0.000 \\
\hline 1 & 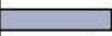 & $\square$ & 1 & 2 & 0.844 & -0.346 & 194.07 & 0.000 \\
\hline 1 & $\square$ & $\square$ & 1 & 3 & 0.720 & -0.228 & 257.93 & 0.000 \\
\hline I & 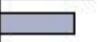 & 문 & 1 & 4 & 0.576 & -0.168 & 299.10 & 0.000 \\
\hline 1 & $\square$ & 10 & 1 & 5 & 0.423 & -0.086 & 321.50 & 0.000 \\
\hline 1 & $\square$ & 1 & 1 & 6 & 0.276 & 0.009 & 331.15 & 0.000 \\
\hline 1 & إ & 10 & 1 & 7 & 0.134 & -0.094 & 333.44 & 0.000 \\
\hline I & {$[1$} & 10 & 1 & 8 & 0.000 & -0.077 & 333.44 & 0.000 \\
\hline I미 & 1 & 11 & 1 & 9 & -0.115 & -0.008 & 335.17 & 0.000 \\
\hline$\square$ & 1 & 1 & 1 & 10 & -0.208 & 0.024 & 340.84 & 0.000 \\
\hline$\square$ & 1 & 10 & 1 & 11 & -0.284 & -0.066 & 351.48 & 0.000 \\
\hline$\square$ & 1 & 10 & I & 12 & -0.340 & -0.048 & 366.97 & 0.000 \\
\hline$\square$ & 1 & 10 & 1 & 13 & -0.384 & -0.089 & 386.84 & 0.000 \\
\hline$\square$ & 1 & 10 & 1 & 14 & -0.413 & -0.036 & 410.05 & 0.000 \\
\hline$\square$ & 1 & 1 & 1 & 15 & -0.426 & 0.004 & 434.99 & 0.000 \\
\hline
\end{tabular}

Fonte: Autores (2019)

Em que: Autocorrelation $=$ Autocorrelação; Partial Correlation $=$ Correlação Parcial; AC = coeficiente de autocorrelação; PAC = coeficiente de correlação parcial; Q-Stat = estatística do teste Ljung-Box; Prob. = probabilidade. 
O comportamento dos lags na Figura 2 indica a existência de autocorrelação entre as observações do preço da celulose em atacado. Em relação à estacionariedade, observa-se que o preço médio da celulose em atacado não é estacionário, pois ocorre inversão do comportamento das autocorrelações, permitindo a aplicação do modelo ARIMA ou SARIMA. Na Figura 3, são representados os gráficos das FAC e FACP para o preço da celulose exportada.

\section{Figura 3 - Gráfico da Função de Autocorrelação e Função de Autocorrelação Parcial do preço médio da celulose brasileira exportada no período entre junho de 2008 a março de 2018}

Figure 3 - Autocorrelation and Partial Autocorrelation Functions chart of the average exported Brazilian cellulose price from June 2008 to March 2018

\begin{tabular}{|c|c|c|c|c|c|c|c|c|}
\hline \multicolumn{2}{|c|}{ Autocorrelation } & \multicolumn{3}{|c|}{ Partial Correlation } & $A C$ & PAC & Q-Stat & Prob \\
\hline 1 & 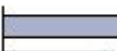 & 1 & & 1 & 0.912 & 0.912 & 100.62 & 0.000 \\
\hline 1 & \begin{tabular}{|c} 
\\
\end{tabular} & 10 & 1 & 2 & 0.815 & -0.096 & 181.76 & 0.000 \\
\hline 1 & 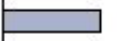 & 10 & 1 & 3 & 0.719 & -0.047 & 245.50 & 0.000 \\
\hline 1 & י & 15 & 1 & 4 & 0.616 & -0.099 & 292.70 & 0.000 \\
\hline 1 & $\square$ & 吅 & 1 & 5 & 0.502 & -0.127 & 324.33 & 0.000 \\
\hline 1 & $\square$ & 10 & 1 & 6 & 0.393 & -0.042 & 343.89 & 0.000 \\
\hline 1 & $\square$ & 1 & ال & 7 & 0.319 & 0.134 & 356.88 & 0.000 \\
\hline 1 & $\square$ & 1 & 1 & 8 & 0.245 & -0.070 & 364.58 & 0.000 \\
\hline 1 & $\square$ & 10 & 1 & 9 & 0.175 & -0.029 & 368.55 & 0.000 \\
\hline 1 & اי & 1 & اך & 10 & 0.136 & 0.109 & 370.98 & 0.000 \\
\hline 1 & اط & 1 & 1 & 11 & 0.107 & -0.026 & 372.49 & 0.000 \\
\hline 1 & 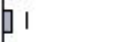 & 1 & 1 & 12 & 0.078 & -0.037 & 373.30 & 0.000 \\
\hline 1 & 51 & 1 & 1 & 13 & 0.056 & 0.024 & 373.72 & 0.000 \\
\hline 1 & 1 & 10 & 1 & 14 & 0.037 & -0.053 & 373.91 & 0.000 \\
\hline 1 & 11 & 10 & 1 & 15 & 0.019 & -0.030 & 373.96 & 0.000 \\
\hline
\end{tabular}

Fonte: Autores (2019)

Em que: Autocorrelation $=$ Autocorrelação; Partial Correlation $=$ Correlação Parcial; $A C$ = coeficiente de autocorrelação; PAC = coeficiente de correlação parcial; Q-Stat = estatística do teste Ljung-Box; Prob. = probabilidade.

A autocorrelação entre as observações confirma-se a partir dos lags significativos presentes nos gráficos (Figura 3). De modo contrário ao preço da celulose em atacado, o preço médio da celulose exportada pode ser considerado estacionário, pois as autocorrelações tornamse não significativas até o lag 12 .

Com o objetivo de confirmar os comportamentos analisados na FAC e FACP, foram realizados os testes de raiz unitária ADF e KPSS. Os resultados dos testes para as duas séries podem ser analisados na Tabela 1.

De acordo com o teste $\mathrm{ADF}$, as duas séries são não estacionárias em nível (d=1); porém, com base nos valores do teste KPSS, as duas séries são estacionárias $(\mathrm{d}=0)$. Logo, os resultados dos testes de raiz unitária contradizem-se e vão de encontro às análises da FAC e FACP. Nessa situação, indica-se a aplicação do modelo fracionário (ARFIMA), pois poderia representar as oscilações dos preços de uma forma mais assertiva e captar o efeito de memória longa presente nas observações (SOUZA et al., 2010; GRAVES et al., 2017). 
Tabela 1 - Testes de raiz unitária para as séries em nível do preço médio da celulose brasileira em atacado e exportada no período entre junho de 2008 e março de 2018

Table 1 - Unit root test with level series of the average price of Brazilian cellulose in wholesale and export from June 2008 to March 2018

\begin{tabular}{lllll}
\hline & \multicolumn{2}{c}{ Preço da celulose em atacado } & \multicolumn{2}{c}{ Preço da celulose exportada } \\
\cline { 2 - 5 } Valor Crítico & ADF & KPSS & ADF & KPSS \\
\hline $\mathbf{1 \%}$ & $-3,4876$ & 0,7390 & $-3,4870$ & 0,7390 \\
$\mathbf{5 \%}$ & $-2,8865$ & 0,4630 & $-2,8863$ & 0,4630 \\
\hline \multirow{2}{*}{ Estatística do teste } & $-2,7176$ & 0,0918 & $-2,2997$ & 0,3102 \\
\hline
\end{tabular}

Fonte: Autores (2019)

Em que: $\mathrm{ADF}=$ Dickey-Fuller Aumentado; KPSS = Kwiatkowski-Phillips-Schmidt-Shin.

Para realizar a modelagem das séries, foram utilizados os modelos da classe geral ARIMA e suas derivações. Os modelos concorrentes, que apresentaram os melhores resultados para os critérios de informação AIC e BIC, e resíduos com característica de Ruído Branco estão descritos nas Tabelas 2 e 3, juntamente com as respectivas estatísticas de previsão (RMSE, MAPE e U-Theil).

Tabela 2 - Modelos ajustados para o preço médio da celulose brasileira em atacado

Table 2 - Adjusted models for the average price of wholesale Brazilian cellulose

\begin{tabular}{|c|c|c|c|c|c|c|c|}
\hline Modelo & Coeficientes & p-valor & AIC & BIC & RMSE & MAPE & U-Theil \\
\hline $\operatorname{ARIMA~}(\mathbf{1 , 1 , 0 )}$ & $\varphi_{1}=0,714130$ & $<0,05$ & 8,556213 & 8,60343 & 17,17174 & 1,476664 & 0,011093 \\
\hline \multirow{3}{*}{$\operatorname{ARIMA}(\mathbf{1 , 1 , 2 )}$} & $\varphi_{3}=0,341182$ & $<0,05$ & \multirow{3}{*}{8,573937} & \multirow{3}{*}{8,668371} & \multirow{3}{*}{17,17741} & \multirow{3}{*}{1,490243} & \multirow{3}{*}{0,011116} \\
\hline & $\theta_{1}=0,707151$ & $<0,05$ & & & & & \\
\hline & $\theta_{2}=0,594134$ & $<0,05$ & & & & & \\
\hline \multirow{4}{*}{$\operatorname{ARFIMA}\left(1, d^{*}, 2\right)$} & $d^{*}=0,491344$ & $<0,05$ & \multirow{4}{*}{8,648579} & \multirow{4}{*}{8,765981} & \multirow{4}{*}{67,45539} & \multirow{4}{*}{8,36959} & \multirow{4}{*}{0,045432} \\
\hline & $\varphi_{1}=0,876619$ & $<0,05$ & & & & & \\
\hline & $\theta_{1}=0,290938$ & $<0,05$ & & & & & \\
\hline & $\theta_{2}=0,214295$ & $<0,05$ & & & & & \\
\hline
\end{tabular}

Fonte: Autores (2019)

Em que: $\mathrm{d}^{*}=$ diferença fracionária; $\varphi=$ parâmetro autorregressivo; $\theta$ = parâmetro de médias móveis; AIC = critério de informação Akaike; BIC = critério de informação Bayesiano; RMSE = Raiz do Erro Quadrático Médio; MAPE = Erro Percentual Médio Absoluto.

Observa-se que todos os parâmetros são significativos, com p-valor inferior a 5\%. Conforme a Tabela 2, o melhor modelo ajustado para o preço médio da celulose em atacado foi o ARIMA $(1,1,0)$, o qual também apresentou os menores valores das estatísticas de previsão (RMSE, MAPE e U-Theil). Com base na Tabela 3, o melhor modelo de previsão para o preço da celulose exportada foi o ARFIMAX $\left(1, d^{*}, 0\right)$, por integrar a diferenciação fracionária e os efeitos de uma variável externa ao modelo $\left(P C_{t-2}\right)$. 
Tabela 3 - Modelos ajustados para o preço médio da celulose brasileira exportada

Table 3 - Adjusted models for the average price of exported Brazilian cellulose

\begin{tabular}{|c|c|c|c|c|c|c|c|}
\hline Modelo & Coeficientes & p-valor & AIC & BIC & RMSE & MAPE & U-Theil \\
\hline \multirow{3}{*}{$\operatorname{ARFIMAX}\left(\mathbf{1}, d^{*}, \mathbf{0}\right)$} & $d^{*}=0,494212$ & $<0,05$ & \multirow{3}{*}{9,319787} & \multirow{3}{*}{9,414739} & \multirow{3}{*}{24,22819} & \multirow{3}{*}{3,866562} & \multirow{3}{*}{0,024632} \\
\hline & $\varphi_{1}=0,397836$ & $<0,05$ & & & & & \\
\hline & $\mathrm{PC}_{\mathrm{t}-2}=0,367492$ & $<0,05$ & & & & & \\
\hline \multirow{3}{*}{$\operatorname{ARIMA}(2,0,0)_{\mathrm{C}}$} & $C=491,4469$ & $<0,05$ & \multirow{3}{*}{9,326563} & \multirow{3}{*}{9,420484} & \multirow{3}{*}{24,78292} & \multirow{3}{*}{3,721748} & \multirow{3}{*}{0,025405} \\
\hline & $\varphi_{1}=0,966837$ & $<0,05$ & & & & & \\
\hline & $\varphi_{6}=-0,092599$ & $<0,05$ & & & & & \\
\hline \multirow{3}{*}{$\operatorname{ARFIMA}\left(\mathbf{1}, d^{*}, \mathbf{1}\right)$} & $d^{*}=0,496067$ & $<0,05$ & \multirow{3}{*}{9,417486} & \multirow{3}{*}{9,511417} & \multirow{3}{*}{157,1302} & \multirow{3}{*}{31,51193} & \multirow{3}{*}{0,189905} \\
\hline & $\varphi_{1}=0,7660802$ & $<0,05$ & & & & & \\
\hline & $\theta_{1}=-0,248343$ & $<0,05$ & & & & & \\
\hline
\end{tabular}

Fonte: Autores (2019)

Em que: $d^{*}=$ diferença fracionária; $\varphi=$ parâmetro autorregressivo; $\theta=$ parâmetro de médias móveis; $P C_{t-2}=$ preço da celulose brasileira em atacado com duas defasagens; $C$ = constante; AIC = critério de informação Akaike; BIC = critério de informação Bayesiano; RMSE = Raiz do Erro Quadrático Médio; MAPE = Erro Percentual Médio Absoluto.

Para verificar a acurácia do ajuste da modelagem, foram elaborados os gráficos que representam os valores ajustados e os resíduos dos modelos escolhidos para cada série do preço da celulose, apresentados nas Figuras 4 e 5.

Figura 4 - Valores reais, ajustados e resíduos do modelo ARIMA (1,1,0), ajustado para o preço médio da celulose brasileira em atacado

Figure 4 - Real, adjusted values and residues of ARIMA model $(1,1,0)$ adjusted for the average price of wholesale Brazilian cellulose

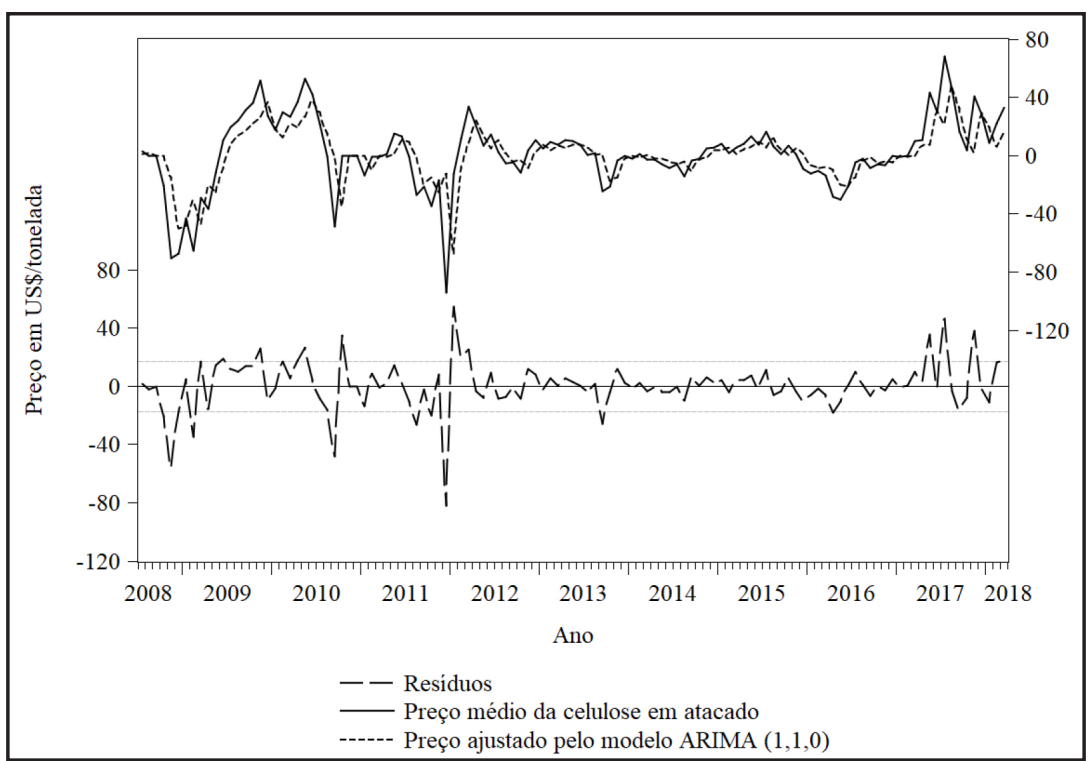

Fonte: Autores (2019) 
A partir da Figura 4, observa-se que o modelo ARIMA $(1,1,0)$ foi capaz de acompanhar as oscilações da série original e, assim, pode ser utilizado para realizar previsões desses comportamentos. A Figura 5 representa o gráfico da modelagem para o preço da celulose brasileira exportada.

Figura 5 - Valores reais, ajustados e resíduos do modelo $\operatorname{ARFIMAX}\left(1, d^{*}, 0\right)$, ajustado para o preço médio da celulose brasileira exportada

Figure 5 - Real, adjusted values and residues of ARFIMAX model $\left(1, d^{*}, 0\right)$ adjusted for the average price of exported Brazilian cellulose

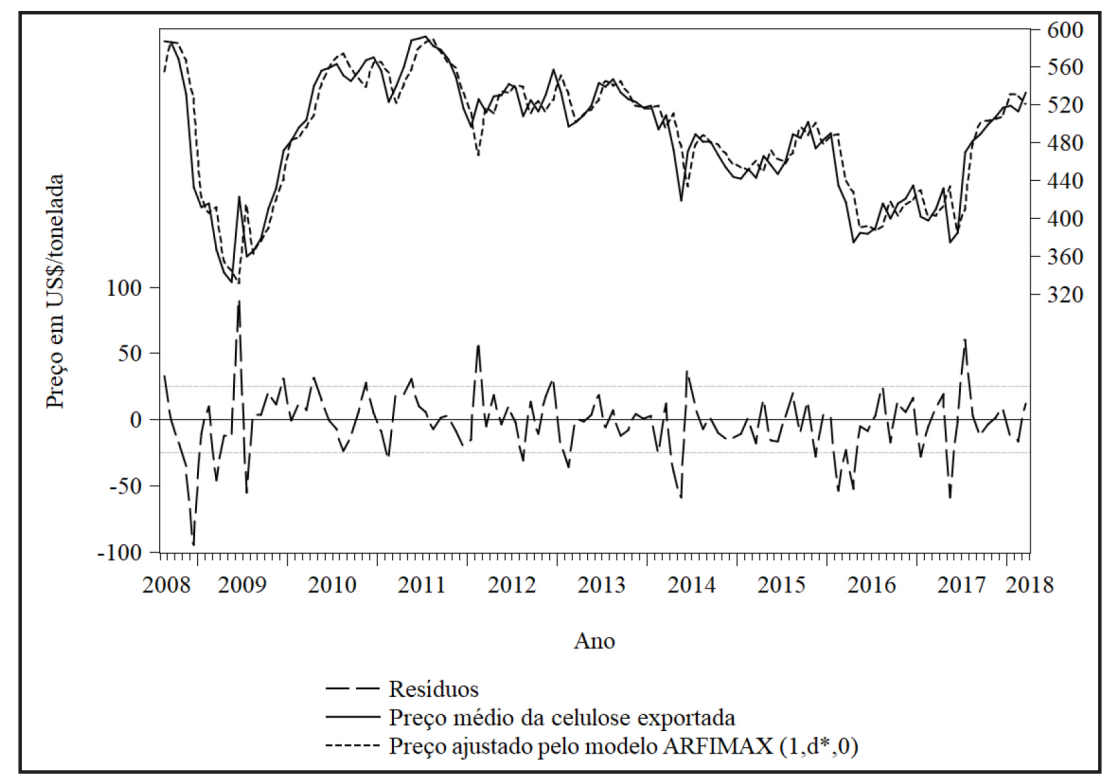

Fonte: Autores (2019)

O modelo ARFIMAX $\left(1, d^{*}, 0\right)$ também se mostrou capaz de acompanhar as variações da série original, indicando que esse modelo pode ser utilizado para prognosticar o preço da celulose exportada (Figura 5).

Em relação aos resíduos, verifica-se que apresentam média em torno de zero, porém existem picos em períodos nos quais as séries originais apresentaram uma mudança abrupta de comportamento (Figuras 4 e 5). Essa característica pode indicar a presença de quebra estrutural nas séries, influenciada por alguma crise econômica, cotação do dólar ou até por políticas governamentais (GONG; LIN, 2018).

De modo a verificar a existência de inter-relações entre o preço da celulose em atacado e o preço da celulose exportada, foi ajustado um modelo VAR. Para o ajuste das equações simultâneas, foram utilizadas as séries diferenciadas devido ao seu comportamento não estacionário.

O número de lags foi definido com base nas estatísticas Erro Final de Previsão (FPE), na estatística sequenciada do teste LR modificado e nos critérios de informação AIC, BIC e HannanQuinn (HQ). A ordem de lag selecionada foi igual a um. As seguintes equações representam o modelo VAR(1) ajustado:

$$
\begin{aligned}
& \Delta P C E_{t}=0,47 \Delta P C_{t-1}-0,15 \Delta P C E_{t-1}-0,92 C+\alpha_{t} \\
& \Delta P C_{t}=0,66 \Delta P C_{t-1}+0,11 \Delta P C E_{t-1}+0,70 C+\alpha_{t}
\end{aligned}
$$

Em que: $\Delta P C_{t}=$ preço da celulose em atacado em primeiras diferenças no período $t ; \Delta P C E_{t}=$ preço da celulose exportada em primeiras diferenças no período $t ; C=$ constante; $\alpha_{t}=$ inovações do modelo; $t=$ tempo. 
Com base no modelo VAR (1), verificou-se que a interferência maior é dos valores do preço da celulose em atacado em relação ao preço da celulose exportada. Essa interferência pode ser visualizada no gráfico da Função Impulso Resposta (Figura 6).

Figura 6 - Gráfico da função de impulso resposta às inovações do modelo VAR (1)

Figure 6 - Impulse response function chart to innovations in the VAR (1) model

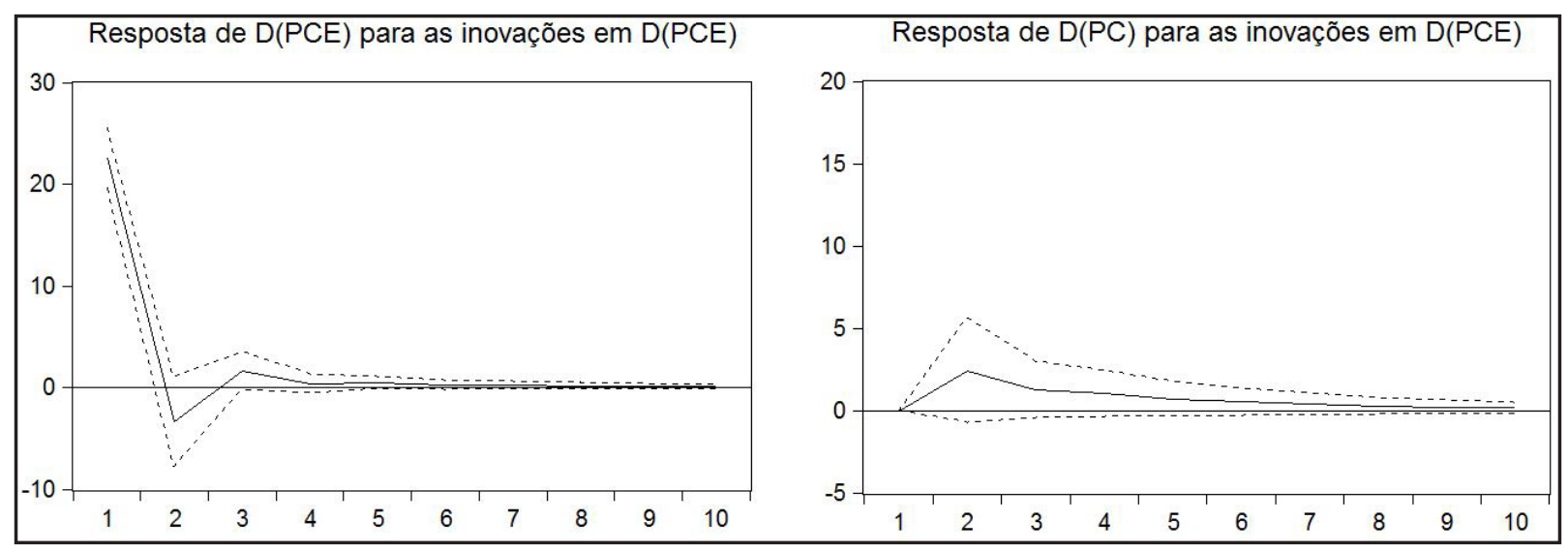

Fonte: Autores (2019)

Em que: $\mathrm{D}(\mathrm{PC})$ = preço da celulose brasileira em atacado em primeiras diferenças; $\mathrm{D}(\mathrm{PCE})$ = preço da celulose brasileira exportada em primeiras diferenças.

Na Figura 6, observa-se que o preço da celulose em atacado estabiliza após o nono período ao receber um impulso do preço da celulose exportada. De modo contrário, quando analisada a resposta do preço da celulose exportada ao impulso do preço da celulose em atacado, observa-se que a estabilidade é alcançada após o décimo primeiro período, porém a amplitude de oscilação é maior.

Essa reação transparece o forte impacto que o preço da celulose em atacado exerce sobre o preço da celulose exportada e a inter-relação positiva entre as variáveis. A influência da celulose nacional nas exportações pode ser evidenciada pelo aumento da competitividade da celulose brasileira no cenário mundial (COSTA et al., 2018).

O retardamento da estabilidade do preço da celulose exportada pode ser justificado pelo impacto que o baixo preço de exportação da celulose exerce sobre o desempenho financeiro das empresas papeleiras, diminuindo seu lucro e valor de mercado (INVESTING, 2019). Outro fator que também impacta a estabilidade do preço da celulose exportada é a variação do nível das exportações, uma vez que o crescimento econômico brasileiro está diretamente atrelado às exportações de commodities primárias (MURAKAMI; HERNÁNDEZ, 2018).

De modo a investigar a variação do preço da celulose exportada causada pelo preço da celulose em atacado, foi realizada a análise da Decomposição da Variância. Os resultados para os períodos 1, 5 e 10 podem ser observados na Tabela 4. 
Tabela 4 - Análise de decomposição da variância do preço da celulose brasileira em atacado em primeiras diferenças e do preço da celulose brasileira exportada em primeiras diferenças

Table 4 - Analysis of variance decomposition of the price of wholesale Brazilian cellulose in its first differences and the price of exported Brazilian cellulose in its first differences

\begin{tabular}{llll}
\hline \multicolumn{4}{c}{ Decomposição da Variância de $\Delta$ PC } \\
\hline Período & Erro & $\mathbf{D}(\mathbf{P C})$ & $\mathbf{D}(\mathbf{P C E})$ \\
\hline $\mathbf{1}$ & 17,21 & 100,00 & 0,00 \\
$\mathbf{5}$ & 24,52 & 98,51 & 1,49 \\
$\mathbf{1 0}$ & 25,01 & 98,48 & 1,52 \\
\hline & Decomposição da Variância de $\Delta$ PCE & \\
\hline Período & Erro & $\mathbf{D}(\mathbf{P C})$ & $\mathbf{D}(\mathbf{P C E})$ \\
\hline $\mathbf{1}$ & 23,90 & 10,64 & 89,36 \\
$\mathbf{5}$ & 25,99 & 22,33 & 77,67 \\
$\mathbf{1 0}$ & 26,13 & 23,11 & 76,89 \\
\hline
\end{tabular}

Fonte: Autores (2019)

Em que: $\mathrm{D}(\mathrm{PC})$ = preço da celulose brasileira em atacado em primeiras diferenças; $\mathrm{D}(\mathrm{PCE})=$ preço da celulose brasileira exportada em primeiras diferenças.

A partir da Tabela 4, observa-se que o preço da celulose em atacado é autoexplicativo no primeiro período e é explicada pelo preço da celulose exportada em $1,52 \%$ no décimo período. Ao analisar o preço da celulose exportada, a mesma é influenciada pela celulose em atacado em $10,64 \%$ e $23,11 \%$, no primeiro e décimo período respectivamente. Os resultados da Decomposição da Variância validam a conclusão da modelagem VAR (1) e dos impulsos de reposta, uma vez que é possível verificar que o impacto do preço da celulose em atacado influencia a variação do preço da celulose exportada de maneira mais significativa com o passar do tempo.

\section{Conclusões}

Ao realizar as previsões dos preços da celulose nos mercados interno e externo, verificouse que os valores possuem oscilações em períodos semelhantes, dado que as variações em índices econômicos influenciam da mesma forma esses mercados. Também se constatou que o preço da celulose exportada é menor que o preço da celulose em atacado, uma vez que o preço é fator indispensável para manter a competitividade da celulose brasileira frente ao cenário mundial.

Ademais, foi identificada a existência de inter-relações entre as variáveis, por meio da modelagem VAR. As interações transpareceram o forte impacto que o preço da celulose em atacado exerce sobre o preço da celulose exportada, apresentando uma interferência positiva entre as variáveis.

Dessa forma, além dos contratos de exportação e da cotação do dólar, o preço da celulose exportada também é influenciado pelo preço da celulose comercializada em atacado nacional. Portanto, conhecer e prognosticar o comportamento do preço da celulose é uma informação importante para as empresas papeleiras, pois o preço impacta diretamente no lucro operacional e no nível de exportações do produto.

Sugere-se para estudos futuros realizar a previsão do preço da celulose com base nos seus principais influenciadores, como cotação do dólar e indicadores financeiros dos países 
importadores, além de analisar a presença de quebras estruturais nas séries dos preços.

\section{Agradecimentos}

À Comissão de Aperfeiçoamento de Pessoal do Nível Superior (CAPES) pelo aporte financeiro, ao Laboratório de Análise e Modelagem Estatística (LAME) da Universidade Federal de Santa Maria pelo espaço utilizado e aos revisores anônimos pelas sugestões realizadas para a melhoria do estudo.

\section{Referências}

AKAIKE, H. A new look at the statistical model identification. IEEE Transactions on Automatic Control, Texas, v. 19, n. 6, p. 716-723, dec. 1974.

BATISTA, F. Títulos da Suzano acompanham baixa dos preços da celulose. São Paulo: Economia UOL, 2019. Disponível em: https://economia.uol.com.br/noticias/bloomberg/2019/09/19/titulosda-suzano-acompanham-baixa-dos-precos-da-celulose.htm. Acesso em: 20 fev. 2020.

BOZDOGAN, H. Model selection and Akaike's information criterion (AIC): the general theory and its analytical extensions. Psychometrika, New York, n. 52, p. 345-370, 1987.

BOX, G. E. P.; JENKINS, G. M. Time series analysis, forecasting and control. San Francisco: Holden Day, 1970.

BOX, G. E. P.; JENKINS, G. M.; REINSEL, G. C. Time series analysis: forecasting and control. 3th ed. New Jersey: Printice Hall, 1994.

BRASIL. Ministério da Agricultura, Pecuária e Abastecimento. Projeções do agronegócio: Brasil 2017/18 a 2027/28 projeções de longo prazo. Brasília, 2018. Disponível em: http://www. agricultura.gov.br/assuntos/politica-agricola/todas-publicacoes-de-politica-agricola/projecoesdo-agronegocio/PROJECOES2018_FINALIZADA_web_05092018.pdf. Acesso em: 10 dez. 2019.

BUENO, R. de L. da S. Econometria de séries temporais. 2. ed. São Paulo: Cengage Learning, 2015.

CELUlOSE ONLINE. As 10 Maiores Fábricas Integradas de Papel no Brasil. [S. l.], 2017. Disponível em: https://www.celuloseonline.com.br/as-10-maiores-fabricas-integradas-de-papelno-brasil-acesso/. Acesso em 05 nov. 2019.

CENTRO DE ESTUDOS AVANÇADOS EM ECONOMIA APLICADA. Florestal. Piracicaba, 2018. Disponível em: https://www.cepea.esalq.usp.br/br/categoria/florestal.aspx. Acesso em: 07 jun. 2018.

CHAREMZA, W.; DEADMAN, D. New directions in econometric practice: general to specific modelling, cointegration and vector autoregression. Cheltenham: Edward Elgar Publishing, 1997.

CORREA, D. V. G. Celulose: logística e distribuição. São Paulo: SENAI-SP, 2014. Disponível em: https://books.google.com.br/books?hl=ptBR\&lr=\&id=Pg5QDwAAQBAJ\&oi=fnd\&pg

$=$ PT6\&dq=Celulose\&ots=pidVZvq0nG\&sig=5PjEav_xtLhQjmxtSebKFNAHhsU\#v=onepage\&q\& $\mathrm{f}=$ false. Acesso em: 25 jun. 2018.

COSTA, T. R. et al. Dinâmica da concentração das exportações brasileiras de celulose química (1990 - 2010). Ciência Florestal, Santa Maria, v. 28, n. 4, p. 1666-1675, 2018.

DICKEY, D. A.; FULLER, W. A. Likelihood ratio statistics for autoregressive time series with a unit root. Econometrica, United Kingdom, v. 49, n. 4, p. 1057-1072, 1981. 
ERSEN, N.; AKYÜZ, I.; BAYRAM, B. Ç. The forecasting of the exports and imports of paper and paper products in Turkey using Box-Jenkins method. Eurasian Journal of Forest Science, Istanbul, v. 7, n. 1, p. 54-53, 2019.

GONG, X.; LIN, B. Structural changes and out-of-sample prediction of realized range-based variance in the stock market. Physica A: Statistical Mechanics and its Applications, Netherlands, v. 494, p. 27-39, 2018.

GRAVES T. et al. Systematic inference of the long-range dependence and heavy-tail distribution parameters of ARFIMA models. Physica A: Statistical Mechanics and its Applications, Netherlands, v. 473, p. 60-71, 2017.

GUSTIN, M.; MCLEOD, R. S.; LOMAS, K. J. Forecasting indoor temperatures during heatwaves using time series models. Building and Environment, United Kingdom, v. 143, p. 727-739, 2018.

HAMILTON, J. Time series analysis. 1st ed. Princeton: Princeton University Press, 1994.

HORA, A. B. da; VIDAL, A. C. F. Panorama setorial 2015-2018: celulose. [S. l.]: Banco Nacional de Desenvolvimento Econômico e Social, 2014. Disponível em: https://web.bndes.gov.br/bib/ jspui/bitstream/1408/14156/1/Celulose_P_BD.pdf. Acesso em: 25 jun. 2018.

HORA, A. B. da; RIBEIRO, L. B. N. M.; MENDES, R. Papel e celulose = Paper and cellulose. In: PUGA, F. P.; CASTRO, L. B. de (org.). Visão 2035: Brasil, país desenvolvido: agendas setoriais para alcance da meta. 1. ed. Rio de Janeiro: Banco Nacional de Desenvolvimento Econômico e Social, 2018, p. 119-142. Disponível em: https://web.bndes.gov.br/bib/jspui/handle/1408/16222. Acesso em: 20 fev. 2020.

INDÚSTRIA BRASILEIRA DE ÁRVORES. Dados Estatísticos. Brasília, 2018. Disponível em: https://iba.org/dados-estatisticos. Acesso em: 05 nov. 2019.

INVESTING. Suzano e Klabin operam com perdas com preço da celulose em queda. [S. l.], 2019. Disponível em: https://economia.uol.com.br/noticias/investing/2019/07/23/suzano-e-klabinoperam-com-perdas-com-preco-da-celulose-em-queda.htm. Acesso em: 20 fev. 2020.

KWIATKOWSKI, D. et al. Testing the null hypothesis of stationarity against the alternative of a unit root. Journal of Econometrics, Netherlands, v. 54, p. 159-178, 1992.

LÜTKEPOHL, H. Introduction to multiple time series analysis. 2nd ed. New York: Springer, 1991.

MADDALA, G. S. Introduction to econometrics. 2nd ed. New York: Macmillan, 1992.

MARGARIDO, M. A.; TUROLLA, F. A.; FERNANDES, J. M. Analysis of price formation in the international Soybean market: the case of Brazil. Agriculture, São Paulo, v. 47, n. 2, p. 71-85, 2002.

MONTENEGRO, F. S. Spread de crédito no setor de papel e celulose: um estudo da precificação de empresas brasileiras no mercado offshore. 2018. Dissertação (Mestrado em Economia e Finanças) - Fundação Getúlio Vargas, São Paulo, 2018.

MORETTIN, P. A. Econometria financeira: um curso em séries temporais financeira. 3. ed. São Paulo: Blucher, 2016.

MOURA, J. M. de et al. Análise da eficiência energética em segmentos industriais selecionados: segmento celulose e papel. Qualitec-Applus, 2018. Disponível em: http://www.mme.gov. br/documents/10584/105176754/PRODUTO+4_Vpublicacao.pdf/f48424df-30ef-464e-ac90e30d97f41936. Acesso em 10 dez. 2019.

MURAKAMI, Y.; HERNÁNDEZ, R. A. The impacts of China on economic growth: evidence for Brazil, Chile, and Peru. Journal of Post Keynesian Economics, United States, v. 41, n. 3, p. 430$454,2018$. 
OLIVEIRA, A. B.; PEREIRA, J. M.; NASCIMENTO, A. A. Cadeia produtiva de papel e celulose e transformações recentes no sudoeste maranhense. Revista Geografia e Interdisciplinaridade, Grajaú, v. 4, n. 12, p. 135-154, jan. 2018.

O PAPEL. Fusão dá origem a uma das maiores empresas do Brasil. [S. l.], 2019. Disponível em: http://www.revistaopapel.org.br/publicacoes.php?id=2999. Acesso em: 20 fev. 2020.

RAMSER, C. A. de S. et al. The importance of principal components in studying mineral prices using vector autoregressive models: Evidence from the Brazilian economy. Resources Policy, United Kingdom, v. 62, p. 9-21, 2019.

ROSSATO, F. G. F. S. et al. Comparison of revealed comparative advantage indexes with application to trade tendencies of cellulose production from planted forests in Brazil, Canada, China, Sweden, Finland and the United States. Forest Policy and Economics, Netherlands, v. 97, p. 59-66, 2018.

SANTOS, D. R. dos et al. Economic and financial performance of the brazilian pulp and paper industry. International Journal of Advanced Engineering Research and Science, Australia, v. 6, n. 7, p. 622-634, 2019.

SANTOS, G. V. dos; ARAÚJO, A. M. C. \& GITAHY, L. M. C. Trabalho, tecnologia e inovação na indústria de processo contínuo: as transformações na indústria brasileira de celulose de mercado. Revista de Administração da UFSM, Santa Maria, v. 9, n. 3, p. 425-447, jul./set. 2016.

SENNA, V. de; SOUZA, A. M. Assessment of the relationship of government spending on social assistance programs with Brazilian macroeconomic variables. Physica A: Statistical Mechanics and its Applications, Netherlands, v. 462, p. 21-31, 2016.

SOARES, P. R. C. et al. Comportamento sazonal da exportação brasileira de celulose para a China entre 1997 e 2012. Floresta, Curitiba, v. 45, n. 2, p. 251-260, jun. 2015.

SOUZA, A. M. et al. Eletrical energy supply for Rio Grande do Sul, Brazil, using forecast combination of weighted eigenvalues. Gestão da Produção, Operações e Sistemas, Bauru, v. 6, n. 3, p. 23-39, jul./set. 2011.

SOUZA, F. M. Modelos de previsão: aplicações à energia elétrica ARIMA-ARCH-AI e ACP. 1. ed. Curitiba: Appris, 2016.

SOUZA, F. M. et al. Uso de modelo de memória longa: previsão de equipamentos para a agroindústria. Iberoamerican Journal of Industrial Engineering, Florianopolis, v. 2, n. 1, p. 137-153, jul. 2010. 